\title{
Summary of Special Session 3
}

\section{Françoise Genova}

CDS, Observatoire Astronomique, 11 rue de l'Université, F-67000 Strasbourg, France email: genova@astro.u-strasbg.fr

The Virtual Observatory is one of the very few truly global endeavours of astronomy, and IAU General Assemblies are the natural places to present the VO to the community. The three-year time scale of IAU is also well suited to measure progress. At the IAU XXV GA in Sydney, 2003, half of the two-day Joint Discussion 8 on Large Telescopes 8 Virtual Observatories: Visions for the Future was devoted to VO talks: the main goal was to review what was expected from the VO in different disciplines of astronomy; many projects were also presented in the poster sessions. This time the three-day VO Special Session has given an excellent overview of present status of the VO, showing both tremendous progress and the work which remains to be done. Several scientific communities (astronomy, solar and heliospheric physics) are working on VO development, national communities have been organizing themselves in VO projects. Implementation by data centres has begun. Additional technical development is required, and there are still technical challenges. There is also a huge work ahead for providing high quality data and services, but the VO development already has a very positive influence on astronomers' work environment, and more is to come.

VO projects are very diverse, depending on local data holdings, technical and scientific expertise, and also of course on the specific demands of the different funding agencies. IVOA provides a common framework to this increasingly diverse community, in particular interoperability standards, which have to be useful and usable. In the coming years, IVOA will have to adjust its organization to support VO uptake by data centres and user communities, and to gather their feedback from implementation and scientific usage. IVOA also has a role to play in the definition of metadata allowing to use information from data pipelines properly. Knowing the origin, limits, strengths, errors, systematics of data is required, not only for the dissemination of data products, but also for data analysis, complex queries, quality control. Proper metadata also has to be defined for theoretical services. Being able to compare data from different surveys, or modelling results with observations, is another aspect of interoperability.

The VO endeavour is science driven and must remain so, but it faces technical challenges, relevant to interdisciplinary work with the IT community. This requires time and effort, and we have to continue to aim at win-win collaborations, in which the VO provides IT teams with excellent research topics and test-beds, and expects operational solutions from the IT developments. When it comes to implementation, adoption of new technologies has to find a proper balance between risk and sustainability - in other words, new technologies have to be implemented not too early, not too late. Of particular importance is the relation with the computational GRID. The VO is a grid of data and services, which fits well with the 'knowledge grid' concept. Many VO aspects do not require the use of the computational GRID, but some important ones do, e.g. for massive data analysis, or 'Virtual data' creation and storage from theoretical modelling. There is a growing community of GRID astronomers, and willingness to build operational services. A bridge has thus to be built between the VO and the GRID. Because of the world-wide nature of the VO, topics such as Single Sign On and interoperability between the different GRID implementations are especially relevant. 
The diversity of the data and service provider community appeared well in the presentations. Large organizations, motivated in particular by the VO development, move towards provision of 'science-ready' data and enhanced data archives, even for complex data such as interferometry, and in some cases size the opportunity to implement deeper changes to improve data and archive management - this is one of the very positive effects of the VO. New facilities and large projects include the VO concept in their plans. Networking of expertise around large facilities, including instrument teams and PIs of key programs, is certainly worth exploring. Several emerging national projects plan to implement digitized archives of older data, with science usage in mind and thus with a strong emphasis on proper data description, astrometric registration, calibration, etc. Many smaller teams are willing to share their knowledge with the community and to provide specific data and expertise to the VO. New services are emerging, such as theoretical services providing modelling results, or matching models with observations; software suites; data analysis services and algorithms; services dedicated to help solving a well defined science question; ambitious, full data analysis and research environments. The VO is not dictating how to manage archives and services - a thin layer on top of the services translates local parameters into standard ones, with a small overhead on data and service providers. But there is a cost in producing and maintaining quality services, and data centres have to be sufficiently funded. A career path to attract, keep and properly reward scientists working in these fields is also needed. Community support is required (e.g., in advisory committees) for funding and careers. Quality assessment is another open question: 'private' publication without quality assessment by some authority is felt as a risk for VO credibility. IVOA has no vocation at present to be the VO 'quality police', and national projects certainly have a role to play here.

The VO user community also showed up during the meeting. All disciplines are concerned, from users of large surveys to '(not so) old-fashioned stellar spectroscopists' (as one of them showed in an excellent, provocative talk). The VO will help, e.g., for multiwavelength, multi-instrument astronomy, for integration of heterogeneous data, but also for comparizon between observations and modelling results, data analysis, statistical analysis - 'high fidelity statistics', search for diamonds in haystacks, etc. The different user communities have requirements on VO tools, which have to be taken into account by the projects, and are welcome to provide their own VO-enabled tools - the VO projects will not write all the tools. There will be a diversity of VO portals, because of the diversity of science needs. It is likely that most VO science usage will not be VO-enabled end-to-end, but rather that VO-enabled data and tools will be used at many stages of research work. Management of simple and complex workflows, and the possibility to keep track of and 'publish' data analysis paths, are important issues.

Widely used tools have already benefited from the VO development, so many astronomers use the VO without being aware of it. Interoperability between tools, collaboration between projects, are already producing innovative and powerful functionnalities. One important role of the VO projects is to find efficient ways of helping users, in particular on advanced usage of tools - actions targetted on specific domains, and on students and post-docs, would certainly be useful. VO does not mean, in spite of some stereotypes, ready-made, press-button astronomy, and astronomers' skills, knowledge, and critical eye on results, will continue to be the key for high quality science!

The VO has not been starting from scratch, there are expertise and lessons learnt from the on-line archive and service providers, and the community is already accustomed to use on-line resources. But the VO is a change in scale, providing a sustainable framework for service publication and seamless science usage, aiming at becoming part of astronomers' everyday life. 